\title{
AP132 Novel gradient boosting method to identify feature importance for ROSC after Cardiac Arrest (RACA)
}

\section{Anna Bichmann*, Robert Spaight*, Krystyna Isakova*}

'East Midlands Ambulance Service NHS Trust, Lincoln, UK

\section{Clinical Background/ Aim:}

There are various factors affecting Return of Spontaneous Circulation (ROSC) after Cardiac Arrest (CA). There have been evaluated ROSC After Cardiac Arrest (RACA) algorithms published reviewing key factors important for patient outcome and success of resuscitation in the out of hospital environment.

Technological advances allow vast collection of clinical data. The question arises?

\section{What data is available?}

What is the best and statistically robust method for big data analysis?

How can we identify hidden confounding factors to improve patient outcome and care?

Only two research papers published to date use Decision Tree Machine Learning to analyse big data sets. $(1,2)$ Our primary aim was to create a new method for analysing large cardiac arrest registries to identify other determining factors for ROSC rates after cardiac arrest. No papers have integrated our method of analysis for cardiac arrest data.

\section{Method:}

Data set: 15342 patient cardiac arrest records

Data base: East Midlands Ambulance Service (EMAS) cardiac arrest registry based on Utstein Criteria in the UK

Time frame: 2014 to $2017 \rightarrow 3$-year analysis reduces bias for annual variation

Type of analysis: Retrospective analysis

Inclusion: All ages groups who have had a 999 call to EMAS, GCS 3, confirmed CA, Resuscitation attempted

Exclusion criteria Patients not in cardiac arrest, DNAR patients, Cardiac arrest on NHS hospital premises, Cardiac arrest occur in transfer between Trusts, Clear evidence of death or injuries not compatible with life

What made the data special: 143 data columns available per patient including airway data, team constitution, medication applied, route of administration, detailed time, defibrillation, crew composition and training level, community first responder data, etc.

Data cleaning: 6121 eligible patients of all ages Decision to remove patients with missing data: potential risk of bias for analysis

Programme used: Python 3 model for the binary classification with 30 independent categorical features and ROSC at any time as our target variable

\section{CatBoost (Category and Boosting):}

- machine learning library handles categorical (CAT) data automatically based on gradient boosting method being an ensemble algorithm, which is a combination of several decision trees

- this creates an improved model

- error function is a sum of variance, bias and noise

\section{Feature 1}

\section{ROC curve}

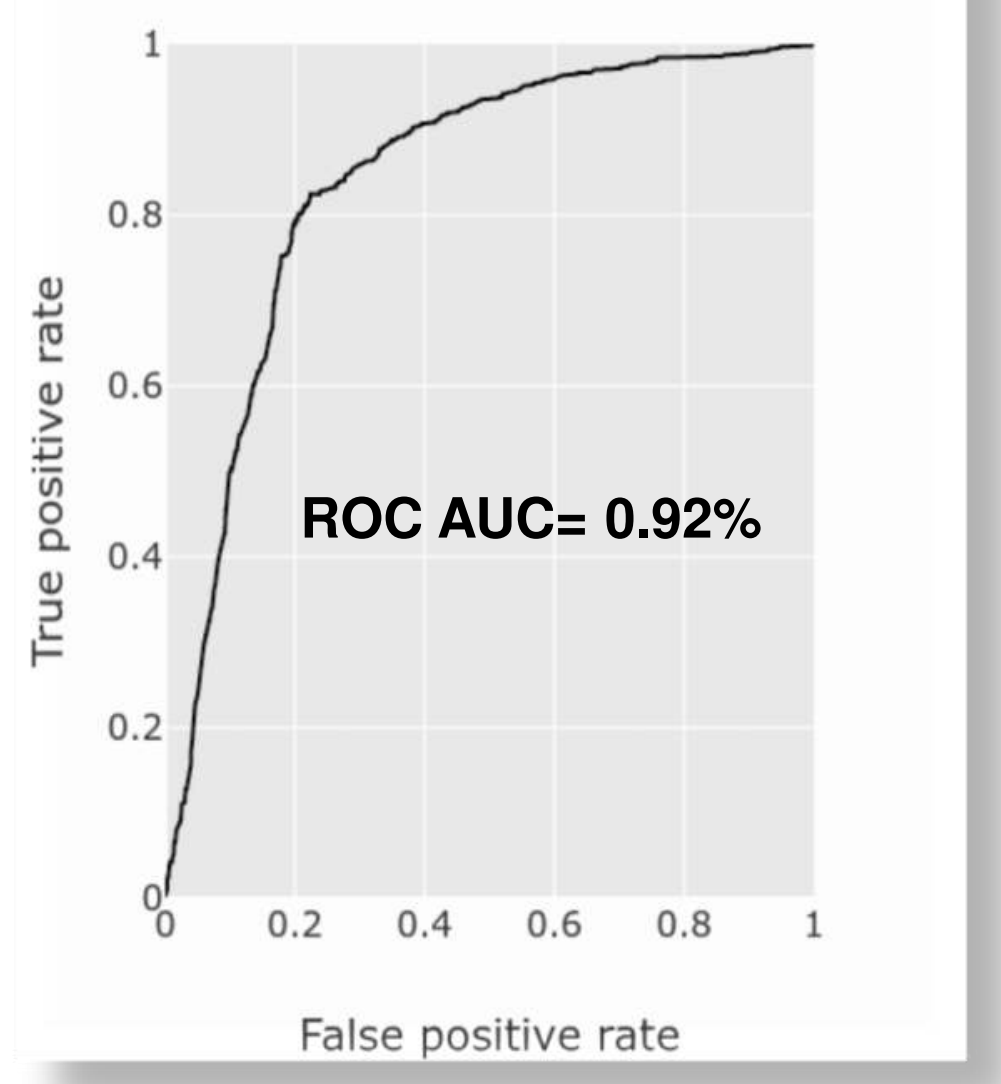

East Midlands Ambulance Service WHS

\section{Feature 2:}

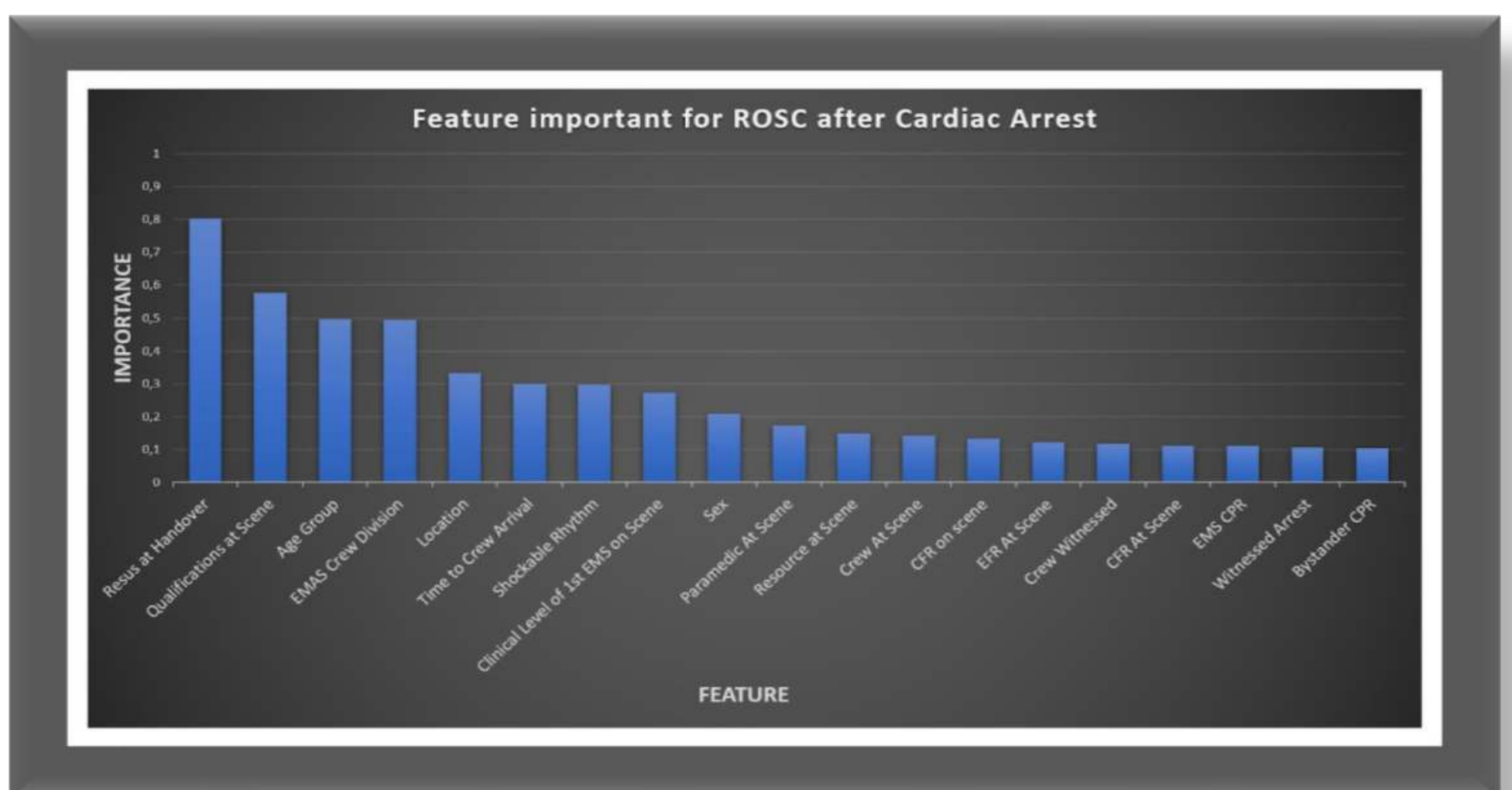

Feature 3:

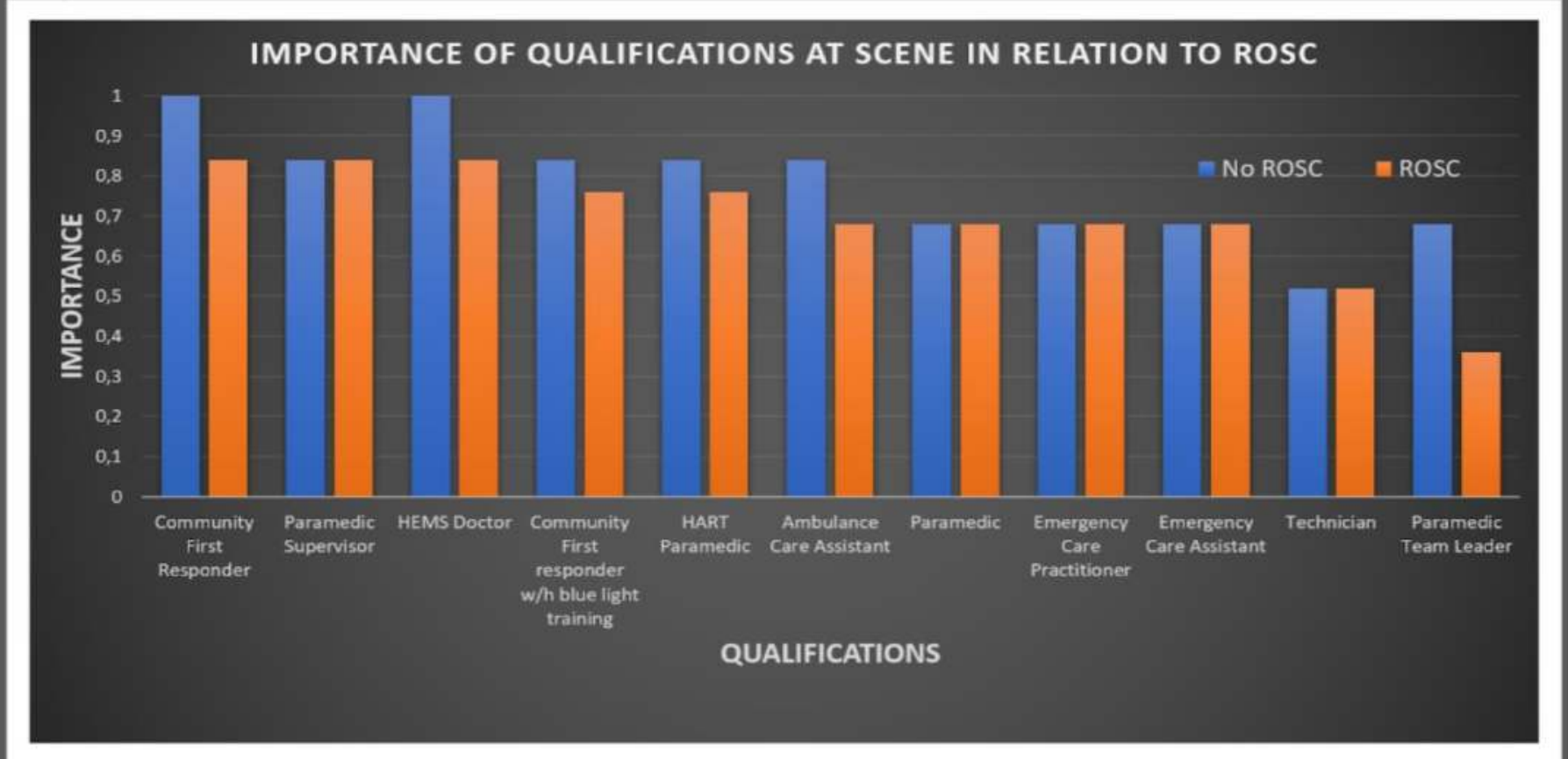

4. Conclusion: We are proposing a new method for CA registries to revalue their own data sets and identify feature importance. This allows extended analysis of CA data and helps ambulance services to critically evaluate their EMS systems

5. Application: EMAS System can use this approach to analyse their clinical data using any outcome variable e.g. Survival, Neurological Outcome and identify previously neglected confounding factors for a targeted system improvement through awareness and training.

6. Limitation: Unidentified and unmeasured confounding factor, No data on quality of life or neurological outcome, One geographical area in the UK, incomplete data sets

\section{References:}

1.Goto Y, Maeda T, Goto Y. Decision-tree model for predicting outcomes after out-ofhospital cardiac arrest in the emergency department. Critical Care, 2013.

2.Goto Y, Maeda T, Goto Y. Decision tree model for predicting long-term outcomes in children with out-of-hospital cardiac arrest: a nationwide, population-based observational study. Critical Care, 2014

\section{Conflict of Interest:}

Financial: No conflict of interests.

Non-financial: Honorary research contract with EMAS, Member of the EPIC Research Group, Warwick 